\title{
Efficiency of Computerized Insulin Infusion Glucose Control in Critically Ill Patients
}

\author{
Hee Jung Lim a, Chi-Min Park a,b,*, Eunmi Gil a,b, Keesang Yoo a , Kyoung-Jin Choi a, Sang-Man Jin c \\ ${ }^{a}$ Department of Surgery, Samsung Medical Center, Sungkyunkwan University School of Medicine, Seoul, Korea \\ ${ }^{b}$ Department of Critical Care Medicine, Samsung Medical Center, Sungkyunkwan University School of Medicine, Seoul, Korea \\ ${ }^{c}$ Division of Endocrinology and Metabolism, Department of Internal Medicine, Samsung Medical Center, Sungkyunkwan University School of Medicine, Seoul, Korea
}

\section{Article history:}

Received: October 23, 2019

Revised: November 8, 2019

Accepted: November 11, 2019

\section{${ }^{*}$ Corresponding Author:}

Chi-Min Park

Department of Surgery and Critical Care Medicine, Samsung Medical Center, Sungkyunkwan University School of Medicine, Seoul, Korea

E-mail:dr99.park@samsung.com

\section{ORCID}

Hee Jung Lim

https://orcid.org/0000-0003-2191-244X

Chi-Min Park

https://orcid.org/0000-0002-8496-3546

Kyoung-Jin Choi

https://orcid.org/0000-0003-2639-2594

\section{Introduction}

Control of blood glucose levels in critically ill patients has been emphasized in ICU management because poor glycemic control increases the risk of adverse outcomes such as nosocomic infection, and morbidity, which would eventually result in increased length of stay and cost of hospital care [1-5]. On the other hand, tight glucose control also increases unwanted events such as severe hypoglycemia which can result in lifelong sequalae [6] and even mortality [7].

Intensive IV insulin infusion therapy has been applied widely for perioperative or critically ill patient care to control glucose levels. However, IV insulin protocols are complex, requiring repeated calculations and skilled nursing care $[4,8,9]$. In practice, blood glucose regulation is controlled using the results from the blood sugar test (BST) according to the specified regular timetable. During the interval between regular BST schedules, it is possible for the patient to become either hyperglycemic or hypoglycemic without showing signs. However, for patients under intensive care, the lack of capacity to overcome the hyper- or hypo-glycemic events proposes a need for a more sophisticated protocol to control blood glucose levels. 
Computerized insulin infusion (CII) protocols have been applied to overcome these issues with the conventional manual insulin infusion protocol. Several computerized protocols have been studied for their efficiency and have been reported to significantly improve the control of blood glucose levels [10-13]. However, there have been few studies conducted on CII protocols for patients under intensive care. Thus, the purpose of this study was to evaluate the safety and efficiency of CII for glucose control in critically ill patients under intensive care unit settings.

\section{Materials and Methods}

This study was conducted in a single medical center (a tertiary university teaching hospital with 1,980 inpatient beds). This institute developed its own CII protocol and integrated it into the hospital's Electronic Medical Record (EMR) order system. The CII protocol was applied to patients whose blood glucose level could not be controlled by the conventional manual protocol. In this study, a total of 132 patients were treated using the CII protocol from July 2016 to February 2017. There were 11 patients excluded because of a low total number of BSTs $(\leq 3)$. Eight patients were excluded due to severe violation of the protocol. Eight patients who received the CII protocol had a different normal target range of blood glucose levels ( $n=2,100-140 \mathrm{mg} / \mathrm{mL} ; n=6,200-299$ $\mathrm{mg} / \mathrm{mL}$ ) to the required normal target range of the study (140$180 \mathrm{mg} / \mathrm{mL}$ ) and were excluded, and 14 patients whose blood glucose level fell within the required normal target range were also excluded. This study was designed to investigate control of glucose levels in critically ill patients under intensive care. Thus, 48 patients on the general wards were also excluded from the study pool. Finally, there were 43 patients in an ICU setting, who were treated using the CII protocol to reach a normal blood glucose level of $70-180 \mathrm{mg} / \mathrm{mL}$.

\section{The CII protocol}

In this study, a CII protocol was used specifically for patients with a blood glucose range of $140-180 \mathrm{mg} / \mathrm{mL}$ to ensure the overall blood glucose level could fall within the targeted normal range $(70-180 \mathrm{mg} / \mathrm{mL}$ ) of this study. A blood glucose level $<70$ $\mathrm{mg} / \mathrm{mL}$ was classified as hypoglycemia, and $>180 \mathrm{mg} / \mathrm{mL}$ was classified as hyperglycemia (severe hyperglycemia $>250 \mathrm{mg} /$ $\mathrm{mL}$ ). When initiating the protocol, the initial insulin infusion rate was determined by the initial blood glucose level measured for each patient. One hour after starting the insulin infusion, the blood glucose level was rechecked and the nurse in charge would input blood glucose level into the EMR order system. The CII protocol automatically calculated the insulin infusion rate and the time interval for the next BST to be performed. In case of severe hyperglycemia (blood glucose level $>250 \mathrm{mg} / \mathrm{mL}$ ) or severe hypoglycemia (blood glucose level $<50 \mathrm{mg} / \mathrm{mL}$ ), the patients were managed according to the guidelines in the protocol. The CII protocol is described in Appendix 1.

The study was approved by the Institutional Review Board of Samsung Medical Center. (IRB file no.: 2019-11-063)

\section{Statistical analysis}

The BST values were obtained throughout the 48 hours prior to, and 48 hours following the implementation of the CII protocol . The primary outcome was the proportion of BSTs in normal target range $(70-180 \mathrm{mg} / \mathrm{mL})$. The statistical analysis was performed using a paired t test when the values followed a standard normal distribution. The Mann-Whitney test was used when the values did not follow a standard normal distribution. $p<0.05$ were considered to indicate statistical significance. All statistics were calculated using IBM SPSS Statistics version 25.

\section{Results}

\section{Baseline characteristics of study subjects}

A total of 43 patients under ICU care were treated with the CII protocol from September 2016 to January 2017 in the Samsung Medical Center, Seoul. Their baseline characteristics are summarized in Table 1 . Their mean age was 63.7 years and their mean BMI was $23.6 \mathrm{~kg} / \mathrm{m} 2$. Thirty patients had a previous history of Type I or Type II diabetes. Twelve patients had not been diagnosed or treated for diabetes prior to hospitalization. Other than newly diagnosed patients, 17 patients had used only oral glucose-lowering medication (39.5\%), and 14 patients had been treated with insulin with or without a combination of oral medications (32.6\%). Their mean HbA1c was 6.6\% (range, 5.10-9.70\%). Their mean serum c-peptide value was $2.01 \mathrm{ng} / \mathrm{mL}$ (range, $0.7-3.6 \mathrm{ng} / \mathrm{mL}$ ).

\section{Frequency of BST measure}

During the 48 hours prior to the implementation of the CII protocol, the total number of BSTs performed was 440, indicating 10.3 /person and $0.4 /$ hour. Following the implementation of the protocol, the number of BSTs performed over 48 hours increased (more than doubled) to 931, indicating 21.7/person $(p<0.001)$ and $0.7 /$ hour $(p<0.001$; Table 2 ). The results demonstrated that by following the CII protocol, BSTs were performed more frequently than before the implementation of the CII protocol.

\section{Glycemic control}

The total number of BST values that fell in the normal target range (70-180 $\mathrm{mg} / \mathrm{mL}$ ) was 99 (22.5\%) before implementing the CII protocol. This number increased significantly to 418 (44.9\%) after following the protocol ( $p<0.001$; Table 3$)$. For severe hyperglycemia control, a blood glucose level over $250 \mathrm{mg} / \mathrm{mL}$ was reported 208 times in the 48 hours prior to implementation 
Table 1. Baseline characteristics of patients.

\begin{tabular}{|ll}
\hline Baseline characteristics & Total $(n=43)$ \\
\hline Age $(\mathrm{y})$ & $63.7(54.00-75.00)$ \\
\hline Gender Male/Female $\mathrm{n} \%)$ & $28(65.1 \%) / 15(34.9 \%)$ \\
\hline BMI $(\mathrm{kg} / \mathrm{m} 2)$ & $23.6(21.1-26.7)$ \\
\hline Duration of diabetes $(\mathrm{y})$ & $8.00(0.00-16.00)$ \\
\hline Type of diabetes & $1(2.3 \%)$ \\
\hline Type 1 & $29(67.4 \%)$ \\
\hline Type 2 & $13(30.2 \%)$ \\
\hline Steroid-induced or post-transplant diabetes & \\
\hline Prior treatment of diabetes & $17(39.5 \%)$ \\
\hline Oral glucose-lowering medication only & $14(32.6 \%)$ \\
\hline Insulin with or without oral medication & $12(27.9 \%)$ \\
\hline No diagnosis or newly diagnosed diabetes & \\
\hline $\begin{array}{l}\text { Chronic kidney disease } \\
\text { (eGRF < 60 mL/min/1.73m2) }\end{array}$ & $13(30.2 \%)$ \\
\hline Reason for IV insulin therapy & $20(46.5 \%)$ \\
\hline NPO for operation & $5(11.6 \%)$ \\
\hline Continuous enteral feeding & $4(9.3 \%)$ \\
\hline Diabetic ketoacidosis & $1(2.3 \%)$ \\
\hline Hyperosmolar hyperglycemic state & $2.01(0.7-3.6)$ \\
\hline C-peptide & \\
\hline
\end{tabular}

BMI = body mass index; eGFR = estimated glomerular filtration rate; $\mathrm{HbA1c}=$ hemoglobin A1c; IV = intravenous; $\mathrm{NPO}=$ non per os.

Table 2. The frequency of BST's measured during the 48 hours prior to, and following the CII protocol implementation.

\begin{tabular}{lccc}
\hline Variables & Pre CII & Post CII & $p$ \\
\hline BST measure (total) & 440 & 931 & \\
BST measure/person & 10.3 & 21.7 & $<0.001$ \\
BST measure/h & 0.4 & 0.7 & $<0.001$ \\
\hline
\end{tabular}

BST = blood sugar test; $\mathrm{CII}=$ computerized insulin infusion.

of the CII protocol (47.3\%). Following implementation of the CII protocol, a BST value over $250 \mathrm{mg} / \mathrm{mL}$ was reported 167 (17.9\%) times out of 931 times in total. This was significantly lower than before the implementation of the CII protocol (47.3\% vs. $17.9 \%$, $p=0.020$ ). Another statistical difference between pre- and postimplementation of the CII protocol was the mean blood glucose level which was $281.4 \mathrm{mg} / \mathrm{mL}$ before the implementation of the CII protocol and $195.5 \mathrm{mg} / \mathrm{mL}$ after the implementation of the CII protocol $(p<0.001)$. There was no significant difference in cases
Table 3. Glycemic variability and the distribution of BST's measured during the 48 hours prior to, and following the CII protocol.

\begin{tabular}{lccc}
\hline Variables & $\begin{array}{c}\text { Pre CII } \\
(n=440)\end{array}$ & $\begin{array}{c}\text { Post CII } \\
(n=931)\end{array}$ & $p$ \\
\hline Within target & $99(22.5 \%)$ & $418(44.9 \%)$ & $<0.001$ \\
$\begin{array}{l}\text { Severe hyperglycemia } \\
(>250)\end{array}$ & $208(47.3 \%)$ & $167(17.9 \%)$ & 0.02 \\
Hypoglycemia & $2(0.45 \%)$ & $1(0.11 \%)$ & 0.239 \\
Mean BG & 281.4 & 195.5 & $<0.001$ \\
\hline $\begin{array}{l}\text { Glycemic variability } \\
\text { SD }\end{array}$ & 68.2 & & \\
CV* & $24.90 \%$ & $26.80 \%$ & 0.622 \\
\hline
\end{tabular}

$\mathrm{BG}=$ blood glucose; $\mathrm{CII}=$ computerized insulin infusion; $\mathrm{CV}=$ coefficient of variation; MGL = Mean Glucose Lever.

${ }^{*} \mathrm{CV}$ of glucose [SD/MGL (\%)].

of hypoglycemia prior to, and following the implementation of the CII protocol ( 2 cases of hypoglycemia pre-implementation and 1 post-implementation of the $\mathrm{CII}$ protocol).

The standard deviation of glycemic variability for the 48 hours prior to implementation of the CII protocol was 68.2 , whilst the 48 hours following the implementation of the CII protocol was 53.3. The SV of the 2 different periods showed a significant difference, suggesting a better glycemic variability control when using the CII protocol. However, the comparison of the coefficient of variation between pre- and post-implementation of the CII protocol indicated this difference was not statistically significant. Figure 1 shows the hourly mean blood glucose levels prior to, and following the implementation of the CII protocol. Comparing trend lines pre- and post-implementation of the CII protocol, the glycemic variability was reduced following implementation of the CII protocol.

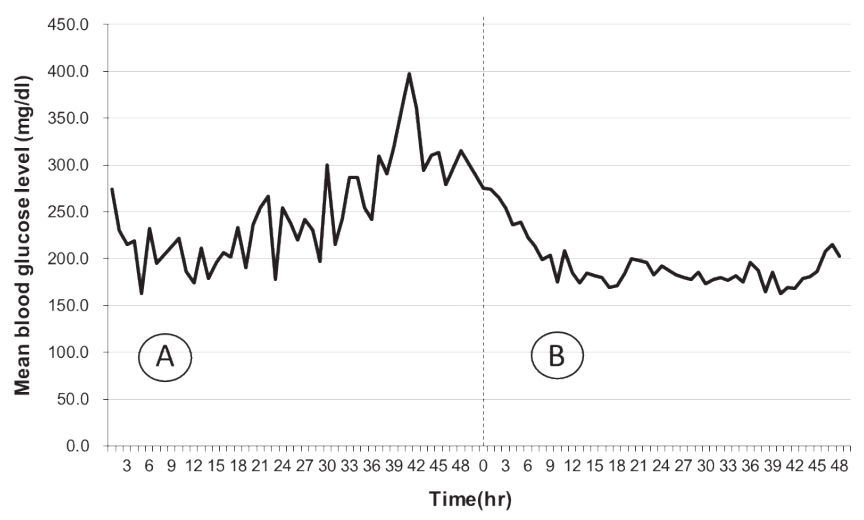

Fig. 1. Hourly mean blood glucose level in (A) pre-computerized insulin infusion implementation period and (B) post-computerized insulin infusion implementation period. 


\section{Discussion}

Management of perioperative blood glucose using a CII protocol leads to a superior optimal glycemic control compared with the standardized insulin infusion protocol $[2,4,8,12,16-18]$. This current study also observed similar results in patients who were critically ill. Blood glucose levels fell within the normal target range $(70-180 \mathrm{mg} / \mathrm{mL})$ more frequently after the implementation of the CII protocol $(p<0.001)$. In addition, severe hyperglycemia events ( $>250 \mathrm{mg} / \mathrm{mL}$ ) were reported significantly less following the implementation of the CII protocol.

When considering optimal blood glucose control, the total number of BSTs was observed to increase following the implementation of the CII protocol. Under the CII protocol setting, the total number of BSTs was 931, whilst the number of BSTs under the conventional continuous blood glucose control setting was 440. Following the continuous insulin infusion protocol, BSTs were performed every 2 hours in the case of hyperglycemia (> $180 \mathrm{mg} / \mathrm{mL}$ ) or hypoglycemia ( $<70 \mathrm{mg} / \mathrm{mL}$ ). However, when using the CII protocol, the intervals between BSTs were between 5 to 15 minutes apart in the case of hypoglycemia. It has been reported that checking blood glucose levels every 2 hours is considered unacceptable for most clinical recommendations [19]. Moreover, the increase in frequency in blood glucose monitoring during the continuous insulin infusion should be interpreted in the context that early stabilization of glucose levels, and early determination of the total insulin requirement would enable earlier initiation of subcutaneous insulin therapy, which requires a substantially lower frequency of BSTs. In fact, the CII protocol used in this study has been shown to enable a more stable control of glucose target levels earlier than the propensity-score matched patients who were studied using the conventional intravenous insulin infusion protocols [9,14-16].

Controlling blood glucose within a normal range is crucial for patients in intensive care units. Some studies have shown significant reductions in mortality and length of ICU stay when blood glucose levels are controlled within a normal range [1-3], emphasizing the benefit of blood glucose control and the need for a better protocol to control it [14]. For a CII to be successful, the protocol must be well integrated into routine medical procedures. In this study, the CII protocol was activated when the physician imputed the a target range for blood glucose levels into the EMR order system. The protocol would then automatically calculate the insulin infusion rate and the next BST . Previous CII studies have used protocol systems which require additional procedures to document in the medical records [12,15]. This double-document system can cause time delays in managing critically ill patients. Thus, the CII protocol used in this study was designed to be integrated into the EMR order system.

The limitation of this study was that only patients who presented with uncontrolled blood glucose levels using the conventional glycemic control protocols were included in the study. In addition, not all these patients were critically ill, which also limited the number of patients included in this study. Moreover, the study data was recorded for only 48 hours of the blood glucose control process. Another limitation was that the CII protocol system used in this study was developed and utilized in a single institute and thus, the patient pool may not be representative of the general population. Lastly, this study compared pre- and post-implementation of the CII protocol in the same group of patients. More researches are required to compare outcomes in the Randomized controlled trials. The computerized delivery system of insulin is currently in practice in the Samsung Medical Center, Sungkyunkwan University, Seoul, and more data for follow-up studies is expected in the near future.

In conclusion, following the CII protocol for glucose control in critically ill patients in the ICU required more frequent BSTs however, resulted in superior control of mean blood glucose levels and glycemic variability, compared with the conventional insulin infusion protocol and significantly decreased hyperglycemia.

\section{Conflicts of Interest}

The authors have no conflicts of interest to declare.

\section{Acknowledgments}

The authors received no specific funding for this work.

\section{References}

[1] Van den Berghe G. Intensive insulin therapy in the ICU-reconciling the evidence. Nat Rev Endocrinol 2012;8(6):374-8.

[2] Van den Berghe G. Intensive insulin therapy to maintain normoglycemia after cardiac surgery. HSR Proc Intensive Care Cardiovasc Anesth 2011;3(2):97-101.

[3] Van den Berghe G, Mesotten D, Vanhorebeek I. Intensive insulin therapy in the intensive care unit. CMAJ 2009;180(8):799-800.

[4] Furnary AP, Zerr KJ, Grunkemeier GL, Starr A. Continuous intravenous insulin infusion reduces the incidence of deep sternal wound infection in diabetic patients after cardiac surgical procedures. Ann Thorac Surg 1999;67(2):352-60.

[5] Krinsley JS. Association between hyperglycemia and increased hospital mortality in a heterogeneous population of critically ill patients. Mayo Clin Proc 2003;78(12):1471-8.

[6] Vriesendorp TM, DeVries JH, van Santen S, Moeniralam HS, de Jonge E, Roos YB, et al. Evaluation of short-term consequences of hypoglycemia in an intensive care unit. Crit Care Med 2006;34(11):2714-8.

[7] Bhatia A, Cadman B, Mackenzie I. Hypoglycemia and cardiac arrest in a critically ill patient on strict glycemic control. Anesth Analg 2006;102(2):549-51.

[8] Taylor BE, Schallom ME, Sona CS, Buchman TG, Boyle WA, Mazuski JE, et al. Efficacy and safety of an insulin infusion protocol in a surgical ICU. J Am Coll Surg 2006;202(1):1-9.

[9] Brown G, Dodek P. Intravenous insulin nomogram improves blood glucose control in the critically ill. Crit Care Med 2001;29(9):1714-9.

[10] Dortch MJ, Mowery NT, Ozdas A, Dossett L, Cao H, Collier B, et al. A computerized insulin infusion titration protocol improves glucose control 
with less hypoglycemia compared to a manual titration protocol in a trauma intensive care unit. JPEN J Parenter Enteral Nutr 2008;32(1):18-27.

[11] Rood E, Bosman RJ, van der Spoel JI, Taylor P, Zandstra DF. Use of a computerized guideline for glucose regulation in the intensive care unit improved both guideline adherence and glucose regulation. J Am Med Inform Assoc 2005;12(2):172-80.

[12] Vogelzang M, Zijlstra F, Nijsten MW. Design and implementation of GRIP: A computerized glucose control system at a surgical intensive care unit. BMC Med Inform Decis Mak 2005;5:38.

[13] Wong B, Mamdani MM, Yu CH. Computerized Insulin Order Sets and Glycemic Control in Hospitalized Patients. Am J Med 2017;130(3):366.e16.

[14] Boutin JM, Gauthier L. Insulin infusion therapy in critically ill patients. Can J Diabetes 2014;38(2):144-50.

[15] Vogelzang M, Loef BG, Regtien JG, van der Horst IC, van Assen H, Zijlstra F, et al. Computer-assisted glucose control in critically ill patients. Intensive
Care Med 2008;34(8):1421-7.

[16] McAlister FA, Man J, Bistritz L, Amad H, Tandon P. Diabetes and coronary artery bypass surgery: An examination of perioperative glycemic control and outcomes. Diabetes Care 2003;26(5):1518-24.

[17] Yendamuri S, Fulda GJ, Tinkoff GH. Admission hyperglycemia as a prognostic indicator in trauma. J Trauma 2003;55(1):33-8.

[18] Ligtenberg JJ, Meertens JH, Monteban-Kooistra WE, Tulleken JE, Zijlstra JG. Multicentric, randomized, controlled trial to evaluate blood glucose control by the model predictive control algorithm versus routine glucose management protocols in intensive care unit patients: Response to Plank et al. Diabetes Care 2006;29(8):1987.

[19] Finfer S, Wernerman J, Preiser J, Cass T, Desaive T, Hovorka R, et al. Clinical Review: Consensus Recommendations on Measurement of Blood Glucose and Reporting Glycemic Control in Critically Ill Adults. Crit Care 2013;17(3):229.

Appendix 1. The Samsung Medical Center computerized insulin protocol designed to achieve a blood glucose range of $140-180 \mathrm{mg} / \mathrm{mL}$.

RI Civ Protocol (target blood glucose range of $140-180 \mathrm{mg} / \mathrm{mL}$ )

(Caution) Only use in case of continuous TPN infusion and at the same rate over 24 hours

(Caution) Only use in case of NPO or continuous EN feeding and at the same rate over 24 hours

1. Mix RI and normal saline to a ratio of 1:2 (ex; RI $50 \mathrm{U}$ in N/S $100 \mathrm{~mL}$ ).

2. Check the initial capillary blood glucose level when starting the RI CIV infusion and recheck the BG level in 1 hour.

$\rightarrow$ input BST check time and blood glucose level information in the EMR.

$\rightarrow$ apply computerized insulin infusion rate.

$\rightarrow$ check the next BG level at calculated time.

3. Initial insulin infusion rate $=($ ) IU/hour: start with integer approximation of (initial BGL/100).

4. In case of a blood glucose level $<70 \mathrm{mg} / \mathrm{mL}$, stop the insulin infusion and inject DW 50\% IV according to the EMR order set on the screen, and notify the doctor in charge.

4.1. If BG level $<50 \mathrm{mg} / \mathrm{mL}$, stop the insulin infusion and inject $50 \mathrm{~mL}$ DW 50\% IV. Repeat the BST in 15 minutes.

4.1.1. Repeat 4.1. until the BG level rises above $110 \mathrm{mg} / \mathrm{mL}$.

4.1.2. 1 hour after discontinuation of the insulin infusion, if the BG level stays above $110 \mathrm{mg} / \mathrm{mL}$, resume insulin infusion.

4.2. In case of a blood glucose level $<50-70 \mathrm{mg} / \mathrm{mL}$, stop the insulin infusion and inject $25 \mathrm{~mL}$ DW $50 \%$ IV. Check the BG level in $5 \mathrm{minutes}$. - If the BG level stays below $70 \mathrm{mg} / \mathrm{mL}$, inject $25 \mathrm{~mL}$ DW 50\% IV, and check BG level in 15 minutes.

4.2.1. Repeat 4.2. until BG level rises above $110 \mathrm{mg} / \mathrm{mL}$.

4.2.2. 1 hour after discontinuation of insulin infusion, if the BG level stays above $110 \mathrm{mg} / \mathrm{mL}$, resume insulin infusion.

$\mathrm{BG}$ = blood glucose; $\mathrm{BGL}=$ blood glucose level; DW = dextrose water; $\mathrm{BST}=$ blood sugar test; $\mathrm{CIV}=$ continuous intravenous; $\mathrm{EMR}=$ electronic medical record; NPO = non per os; $\mathrm{EN}=$ enteral nutrition; N/S = normal saline; $\mathrm{RI}=$ regular insulin; $\mathrm{TPN}=$ total parenteral nutrition. 